\title{
FREE MODULAR LATTICES
}

BY

\section{RALPH FREESE ${ }^{1}$}

\begin{abstract}
It is shown that the word problem for the free modular lattice on five generators is recursively unsolvable.
\end{abstract}

In this paper we show that the word problem for the free modular lattice on five generators, FM(5), is unsolvable. That is, there is no algorithm which can decide for arbitrary lattice terms $u$ and $v$ in five variables if $u=v$ holds identically in all modular lattices. In fact, we show that there is a fixed lattice term $u_{0}$ in five variables such that there is no algorithm for deciding if $u_{0}=v$ holds in all modular lattices for an arbitrary five variable lattice term $v .^{2}$

The free modular lattice on three generators, which is finite, was described in 1900 by R. Dedekind [7] and G. Birkhoff observed that FM(4) was infinite [2]. Interest in the word problem for free modular lattices greatly increased after P. Whitman's solution of the word problem for free lattices appeared [36]. Some partial results were obtained by K. Takeuchi [34], [35] (see Whitman's article [39]). Positive solutions were announced by Schutzenberger [33] and Gluhov [17] but these were refuted by Jonsson (see [38]) and Herrmann [20].

Interest in this problem was renewed in the late sixties partly because the wide applicability of Whitman's results was becoming apparent. Perhaps the most important result was that of G. Hutchinson and, independently, L. Lipshitz that there is a finitely presented modular lattice with unsolvable word problem [24], [41]. Hutchinson went on to show that this presentation could have five generators and one relation [25]. Moreover his results apply to many subvarieties of the variety of all modular lattices.

Certain positive results were also described. R. Wille characterized those partially ordered sets $P$ such that the modular lattice freely generated by $P$ is finite [40]. C. Herrmann and A. Huhn have shown that for certain varieties of modular lattices generated by submodule lattices the word problem for the free lattices in these varieties is solvable [22]. Interestingly there is a nonempty intersection of these varieties and those to which Hutchinson's results apply. In particular the variety $\mathbb{Q}$ generated by all subgroup lattices of abelian groups has a finitely

Received by the editors December 4, 1978 and, in revised form, November 27, 1979; presented to the Society, October 19, 1978 under the title Recent results in modular lattice theory.

AMS (MOS) subject classifications (1970). Primary 06A30; Secondary 02F47.

${ }^{1}$ This research was partially supported by NSF Grant No. MCS77-01933.

${ }^{2}$ The mathematically precise formulation of these results should be in terms of recursive functions [5]. Since our proof reduces the problem to showing that there is a finitely presented group with unsolvable word problem, it is not necessary for us to give a formal definition of a recursive function. 
presented lattice (five generators, one relation) with an unsolvable word problem. However its free lattices have solvable word problems. This explains to some extent why it was difficult to show $\operatorname{FM}(5)$ has an unsolvable word problem. This is discussed more fully in $\S 3$.

There have been many recent results on four-generated modular lattices. Notably C. Herrmann has been able to effectively list all four-generated, subdirectly irreducible modular lattices in the variety $C$ generated by all complemented modular lattices [19]. Hence four-generated finitely presented lattices in $C$ have solvable word problems but not for five generators since Hutchinson's result applies here. Herrmann has also shown that the modular lattice freely generated by two complemented pairs has a solvable word problem [21]. Some of the papers in the references have additional results related to free modular lattices.

The basic idea of our proof is this; associated with each 4-frame (definitions below) in a modular lattice is a ring with identity element. If $R$ is a ring with identity and if $L\left(R^{4}\right)$ is the lattice of submodules of $R^{4}$ as a left $R$-module, then $L\left(R^{4}\right)$ has a 4-frame whose associated ring is $R$. Let $R$ be a ring that contains a finitely presented group with an unsolvable word problem. Let $f$ be a homomorphism from $\operatorname{FM}(X)$ onto $L\left(R^{4}\right)$. It is possible to find a 4-frame in $\operatorname{FM}(X)$ which maps under $f$ to the 4-frame in $L\left(R^{4}\right)$. Unfortunately it is not possible to pull very much of the ring $R$ back through $f$ into $\operatorname{FM}(X)$ (see §3).

In [14] it is shown that the lattice $L$ obtained by gluing $L\left(F^{4}\right)$ and $L\left(K^{4}\right)$ together over a two-dimensional quotient (à la Hall-Dilworth) is not in the variety generated by the finite modular lattices if $F$ and $K$ are countable fields of different prime characteristics. If we now let $f$ be a homomorphism from $\operatorname{FM}(X)$ onto $L$ it is possible to bring back through $f$ those elements of $F$ which are lined up with the prime subfield of $K$ in the gluing. It is now natural to try to glue $L\left(R^{4}\right)$ to $L\left(S^{4}\right)$ in this manner, where $R$ contains a group (or semigroup) with unsolvable word problem and $S$ is some ring. For the ring $R$ used by Hutchinson (the endomorphism ring of an infinite-dimensional vector space) this is not possible, essentially because the automorphisms of $L\left(R^{2}\right)$ come from those of $R$ [31]. However for a countable division ring $D, L\left(D^{2}\right)$ is the two-dimensional lattice with countably many atoms, $M_{\omega}$. Of course this lattice has many automorphisms not induced by $D$. Although it seems unlikely that a group with unsolvable word problem could be embedded into a division ring, A. Macintyre, using some constructions of P. M. Cohn, has shown that if $F$ is a finitely generated free group and $N$ is a finitely generated normal subgroup of $F$ then there is a division ring $D$ containing $F$ and an element $t \in D$ such that if $w \in F$ then $w \in N$ if and only if $w t=t w$ in $D$. Using the lattice $L$ as above with $L\left(D^{4}\right)$ in place of $L\left(F^{4}\right)$ we are able to pull this same situation back into $\mathrm{FM}(5)$, proving that its word problem is unsolvable.

In this paper we use + and juxtaposition for the lattice operations. This simplifies the apparent complexity of the lattice terms and makes it easier for the reader to refer to [14]. For rings interpreted into modular lattices we use $\oplus$ and $\otimes$ for the operations. Other than this, our notation is taken from [4]. In particular for $a \geqslant b \in L, a / b$ denotes the quotient sublattice $\{x \in L: a \geqslant x \geqslant b\}$. If $a+d=c$ 
and $a d=b$ we say that $a / b$ transposes up to $c / d$ and write $a / b \neg c / d$. We also write $c / d \searrow a / b$.

We would like to point out that the proof of our theorem depends on some ingenious results of others. We use John von Neumann's results on coordinates in modular lattices, A. Macintyre and P. M. Cohn's skew field constructions, and the modular lattice-theoretic construction of Marshall Hall and R. P. Dilworth. We would also like to thank Bjarni Jónsson. Our lattice $L$ was originally constructed to solve his innocuous sounding problem "are there uncountable distributive sublattices of free modular lattices?" [28], [14].

1. Preliminaries. A subset $\left\{a_{1}, \ldots, a_{n}, c_{12}, \ldots, c_{1 n}\right\}$ of a modular lattice $L$ is called an $n$-frame if

$$
\begin{gathered}
a_{i}\left(\sum_{\substack{j=1 \\
j \neq i}}^{n} a_{j}\right)=\prod_{j=1}^{n} a_{j}, \quad i=1, \ldots, n, \\
a_{1}+c_{1 i}=a_{i}+c_{1 i}=a_{1}+a_{i}, \quad i=2, \ldots, n, \\
a_{1} c_{1 i}=a_{i} c_{1 i}=a_{1} a_{i}, \quad i=2, \ldots, n .
\end{gathered}
$$

This says that, provided they are distinct, $a_{1}, \ldots, a_{n}$ generates a copy of $2^{n}$ with the $a_{i}$ as atoms and that $\left\{a_{1}, c_{1 i}, a_{i}\right\}$ generates a sublattice isomorphic to $M_{3}$. We let 0 denote $a_{1} a_{2}$ and we do not insist that this is the least element of $L$. Whenever we have an $n$-frame in a modular lattice $L$, define $c_{j 1}=c_{1 j}, j \neq 1$, and for $1, i, j$ distinct $c_{i j}=\left(c_{1 i}+c_{1 j}\right)\left(a_{i}+a_{j}\right)$. By Lemma 5.3 of [31],

$$
c_{i k}=\left(c_{i j}+c_{j k}\right)\left(a_{i}+a_{k}\right)
$$

for distinct $i, j, k$. Let

$$
R=\left\{x \in L: x+a_{2}=a_{1}+a_{2} \text { and } x a_{2}=0\right\}
$$

and define operations $\oplus$ and $\otimes$ on $R$ by

$$
\begin{aligned}
& x \oplus y=\left[\left(x+c_{13}\right)\left(a_{2}+a_{3}\right)+\left(y+a_{3}\right)\left(a_{2}+c_{13}\right)\right]\left(a_{1}+a_{2}\right), \\
& x \otimes y=\left[\left(x+c_{23}\right)\left(a_{1}+a_{3}\right)+\left(y+c_{13}\right)\left(a_{2}+a_{3}\right)\right]\left(a_{1}+a_{2}\right) .
\end{aligned}
$$

The next theorem is essentially von Neumann's Theorem 8.4, p. 157 of [31]. Von Neumann assumed that $L$ was complemented, but his proof of this theorem does not use this. That our definition of $R$ is equivalent to von Neumann's follows easily from Lemma 6.1 of [31].

THEOREM 1.1. Let $\left\{a_{1}, \ldots, a_{n}, c_{12}, \ldots, c_{1 n}\right\}$ be an $n$-frame in a modular lattice $L$, with $n \geqslant 4$. Then $(R, \oplus, \otimes)$ defined above is a ring with unit. The null element of $R$ is $a_{1}$ and $c_{12}$ is the unit.

$$
\text { If } \begin{aligned}
x \in a_{i}+a_{j} / 0 \text { (i.e. } 0 & \left.\leqslant x \leqslant a_{i}+a_{j}\right) \text { then we let } \\
\alpha_{i j}(x) & =\left(\left(x+a_{k}\right)\left(c_{i k}+a_{j}\right)+c_{j k}\right)\left(a_{i}+a_{j}\right) .
\end{aligned}
$$

This definition is independent of the choice of $k$ distinct from $i$ and $j$. Notice that if $x \in R$ then $\alpha_{12}(x)=1 \oplus x,\left(1=c_{12}\right)$. An $n$-frame $\left\{a_{i}, c_{1 j}\right\}$ is called an $n$-frame of characteristic $q$ if $\alpha_{12}^{q}\left(a_{1}\right)=a_{1}$, where $\alpha_{12}^{q}$ is $\alpha_{12}$ iterated $q$ times (cf. [14]). 
LEMMA 1.2. Let $\left\{a_{i}, c_{1 j}\right\}$ be an $n$-frame in a modular lattice $L$, with $n>4$, and let $(R, \oplus, \otimes)$ be the associated ring. An element $x \in R$ has a two-sided multiplicative inverse if and only if $x+a_{1}=a_{1}+a_{2}$ and $x a_{1}=0$. If this is the case then

$$
x^{-1}=\left(\left(\left(x+c_{23}\right)\left(a_{1}+a_{3}\right)+c_{12}\right)\left(a_{2}+a_{3}\right)+c_{13}\right)\left(a_{1}+a_{2}\right) \text {. }
$$

Proof. A proof of the first statement is given in Lemma 2.3 of [15]. The second statement can also be derived from this lemma or alternatively the reader can verify directly that $x \otimes x^{-1}=x^{-1} \otimes x=1\left(=c_{12}\right)$. (Also see p. 158 of [31].)

The following situation will frequently arise. There is an index $k$ with $1<k<n$ and an element $b_{k}$ such that $0 \leqslant b_{k} \leqslant a_{k}$. In this situation we define $b_{1}=$ $\left(b_{k}+c_{1 k}\right) a_{1}$ if $k \neq 1$ and $b_{i}=\left(b_{1}+b_{1 i}\right) a_{i}, i \neq 1, k$, and $b=b_{1}+\cdots+b_{n}$. The reader can check that $b_{k}=\left(b_{1}+c_{1 k}\right) a_{k}$ also holds. It is shown in [23] and Lemma 1.1 of [14] that $\left\{a_{i}+b, c_{1 j}+b\right\}$ is an $n$-frame, whose least element is $b$. Moreover if $\left\{a_{i}, c_{1 j}\right\}$ is an $n$-frame of characteristic $q$ then $\left\{a_{i}+b, c_{1 j}+b\right\}$ is also. Also this frame behaves properly with respect to the other indices. That is

$$
\left(c_{i j}+c_{j k}+b\right)\left(a_{i}+a_{k}+b\right)=\left(c_{i j}+c_{j k}\right)\left(a_{i}+a_{k}\right)+b=c_{i k}+b
$$

In the next four lemmas we assume that $\left\{a_{i}, c_{i j}\right\}$ is an $n$-frame and that $b_{1}, \ldots, b_{k}, b$ are as above. The readers can verify that $b_{i}+c_{i j}=b_{j}+c_{i j}$ for all $i \neq j$. Thus $b_{i} \leqslant b_{j}+c_{i j}$. These facts will be used extensively below.

LEMmA 1.3. If $x \in a_{i}+a_{j} / 0$ satisfies $x+b_{j} \geqslant b_{i}$ then $\alpha_{i j}(x)+b_{j}>b_{i}$ also holds.

Proof.

$$
\begin{aligned}
\alpha_{i j}(x)+b_{j} & =\left(\left(x+a_{k}\right)\left(c_{i k}+a_{j}\right)+c_{j k}\right)\left(a_{i}+a_{j}\right)+b_{j} \\
& =\left(\left(x+b_{j}+a_{k}\right)\left(c_{i k}+a_{j}\right)+c_{j k}\right)\left(a_{i}+a_{j}\right) \\
& =\left[\left(x+b_{i}+b_{j}+a_{k}\right)\left(c_{i k}+a_{j}\right)+c_{j k}\right]\left(a_{i}+a_{j}\right) \\
& =\left(b_{j}+\left(x+b_{i}+a_{k}\right)\left(c_{i k}+a_{j}\right)+c_{j k}\right)\left(a_{i}+a_{j}\right) \\
& \geqslant\left(b_{k}+\left(x+b_{i}+a_{k}\right)\left(c_{i k}+a_{j}\right)\right)\left(a_{i}+a_{j}\right) \\
& =\left(x+b_{i}+a_{k}\right)\left(c_{i k}+b_{k}+a_{j}\right)\left(a_{i}+a_{j}\right)>b_{i} .
\end{aligned}
$$

LEMMA 1.4. Suppose $\left\{a_{i}, c_{1 j}\right\}$ is an $n$-frame of characteristic $q(q \neq 0)$. Let $r$ be invertible in $\mathrm{Z} / \mathrm{qZ}$. Then

$$
\alpha_{i j}^{r}\left(a_{i}\right)+b_{i}=\alpha_{i j}^{r}\left(a_{i}\right)+b_{j}
$$

Proof. Let $r t \equiv 1(\bmod q)$. Then $\alpha_{i j}^{r}\left(a_{i}\right)=\alpha_{j i}^{t}\left(a_{j}\right)$ by (2.11) of [15]. The lemma now follows from two applications of Lemma 1.3.

LEMMA 1.5. Let $R$ be the ring corresponding to the $n$-frame $\left\{a_{i}, c_{1 j}\right\}$. Let $x$ be an invertible element of $R$. If $x+b_{1}=x+b_{2}$ then $x^{-1}+b_{1}=x^{-1}+b_{2}$. 


\section{Proof.}

$$
\begin{aligned}
x^{-1}+b_{2} & =\left(\left(\left(x+c_{23}\right)\left(a_{1}+a_{3}\right)+c_{12}\right)\left(a_{2}+a_{3}\right)+c_{13}\right)\left(a_{1}+a_{2}\right)+b_{2} \\
& =\left(\left(\left(x+c_{23}\right)\left(a_{1}+a_{3}\right)+c_{12}+b_{2}\right)\left(a_{2}+a_{3}\right)+c_{13}\right)\left(a_{1}+a_{2}\right) \\
& =\left(\left(\left(x+c_{23}\right)\left(a_{1}+a_{3}\right)+c_{12}+b_{1}\right)\left(a_{2}+a_{3}\right)+c_{13}\right)\left(a_{1}+a_{2}\right) \\
& =\left(\left(\left(x+b_{1}+c_{23}\right)\left(a_{1}+a_{3}\right)+c_{12}\right)\left(a_{2}+a_{3}\right)+c_{13}\right)\left(a_{1}+a_{2}\right) \\
& =\left(\left(\left(x+b_{2}+c_{23}\right)\left(a_{1}+a_{3}\right)+c_{12}\right)\left(a_{2}+a_{3}\right)+c_{13}\right)\left(a_{1}+a_{2}\right) \\
& =\left(\left(\left(x+b_{3}+c_{23}\right)\left(a_{1}+a_{3}\right)+c_{12}\right)\left(a_{2}+a_{3}\right)+c_{13}\right)\left(a_{1}+a_{2}\right) \\
& =\left(\left(\left(x+c_{23}\right)\left(a_{1}+a_{3}\right)+c_{12}+b_{3}\right)\left(a_{2}+a_{3}\right)+c_{13}\right)\left(a_{1}+a_{2}\right) \\
& =\left(\left(\left(x+c_{23}\right)\left(a_{1}+a_{3}\right)+c_{12}\right)\left(a_{2}+a_{3}\right)+b_{3}+c_{13}\right)\left(a_{1}+a_{2}\right) \\
& =\left(\left(\left(x+c_{23}\right)\left(a_{1}+a_{3}\right)+c_{12}\right)\left(a_{2}+a_{3}\right)+b_{1}+c_{13}\right)\left(a_{1}+a_{2}\right) \\
& =x^{-1}+b_{1} .
\end{aligned}
$$

We denote the ring corresponding to the frame $\left\{a_{i}+b, c_{i j}+b\right\}$ by $R(b)$. Thus $R(b)=\left\{x \in L: x+a_{2}+b=a_{1}+a_{2}+b\right.$ and $x\left(a_{1}+b\right)=\left(a_{1}+b\right)\left(a_{2}+b\right)=$ $b$ \}. The operations $\oplus$ and $\otimes$ in $R(b)$ are obtained from (1.6) and (1.7) by substituting $a_{i}+b$ for $a_{i}$ and $c_{i j}+b$ for $c_{i j}$.

LEMMA 1.6. Let $z_{1}, \ldots, z_{m}$ be invertible elements of $R$ and suppose $z_{i}+b_{1}=z_{i}+$ $b_{2}, i=1, \ldots, m$. Let $w$ be a group theoretic term in $m$ variables. Then $z_{i}+b$ is an invertible element of $R(b)$ and

$$
w\left(z_{1}+b, \ldots, z_{m}+b\right)=w\left(z_{1}, \ldots, z_{m}\right)+b .
$$

Here $w\left(z_{1}+b, \ldots, z_{m}+b\right)$ is evaluated in $R(b)$ and $w\left(z_{1}, \ldots, z_{m}\right)$ is evaluated in R.

Proof. If $w$ is a variable, say $w=z_{i}$, then

$$
\begin{aligned}
\left(z_{i}+b\right)\left(a_{2}+b\right) & =\left(z_{i}+b_{1}+b_{2}+b_{3}+\cdots+b_{n}\right)\left(a_{1}+a_{2}\right) a_{2}+b \\
& =\left[z_{i}+b_{1}+b_{2}+\left(b_{3}+\cdots+b_{n}\right)\left(a_{1}+a_{2}\right)\right] a_{2}+b \\
& =\left(z_{i}+b_{1}+b_{2}\right) a_{2}+b=\left(z_{i}+b_{2}\right) a_{2}+b=z_{i} a_{2}+b_{2}+b=b .
\end{aligned}
$$

This trick of reducing $z_{i}+b$ to $z_{i}+b_{1}+b_{2}$ will be used repeatedly below. From this it follows that $z_{i}+b \in R(b)$. Since $\left(z_{i}+b\right)\left(a_{1}+b\right)=b$ and $z_{i}+b+a_{1}+b$ $=a_{1}+a_{2}+b$ also hold, $z_{i}+b$ is an invertible element of $R(b)$. The following calculations show that the inverse of $z_{i}+b$ in $R(b)$ is the join of $b$ and the inverse of $z_{i}$ in $R$.

$$
\begin{aligned}
&\left(z_{i}+b\right)^{-1}=\left[\left(\left(z_{i}+c_{23}+b\right)\left(a_{1}+a_{3}+b\right)+c_{12}+b\right)\left(a_{2}+a_{3}+b\right)+c_{13}+b\right] \\
& \\
& \cdot\left(a_{1}+a_{2}+b\right) \\
&= {\left[\left(\left(z_{i}+c_{23}+b_{1}+b_{2}+b_{3}\right)\left(a_{1}+a_{3}\right)+c_{12}+b\right)\left(a_{2}+a_{3}\right)+c_{13}+b\right] } \\
& \cdot\left(a_{1}+a_{2}\right)+b \\
&= {\left[\left(\left(z_{i}+c_{23}\right)\left(a_{1}+a_{3}\right)+c_{12}+b_{1}+b_{2}+b_{3}\right)\left(a_{2}+a_{3}\right)+c_{13}+b\right]\left(a_{1}+a_{2}\right)+b } \\
&= {\left[\left(\left(z_{i}+c_{23}\right)\left(a_{1}+a_{3}\right)+c_{12}\right)\left(a_{2}+a_{3}\right)+c_{13}+b_{1}+b_{2}+b_{3}\right]\left(a_{1}+a_{2}\right)+b } \\
&= z_{i}^{-1}+b .
\end{aligned}
$$


Here we have repeatedly used $b_{i} \leqslant b_{j}+c_{i j}$.

Let $y$ be one of the $z_{i}$ or one of their inverses and observe that since $y+b_{1}=y$ $+b_{2}$

$$
\begin{aligned}
\left(y+c_{23}\right)\left(a_{1}+a_{3}\right)+b_{3} & =\left(y+b_{3}+c_{23}\right)\left(a_{1}+a_{3}\right)=\left(y+b_{2}+c_{23}\right)\left(a_{1}+a_{3}\right) \\
& =\left(y+b_{1}+c_{23}\right)\left(a_{1}+a_{3}\right)=\left(y+c_{23}\right)\left(a_{1}+a_{3}\right)+b_{1} .
\end{aligned}
$$

Now, proceeding by induction, let $w=y u$, where $y$ again is a variable or the inverse of a variable. Thus by the inductive hypothesis $u\left(z_{1}+b, \ldots, z_{m}+b\right)=$ $u\left(z_{1}, \ldots, z_{m}\right)+b=u+b$. Thus using the above and $b_{i}<b_{j}+c_{i j}$ we have

$$
\begin{aligned}
w\left(z_{1}+\right. & \left.b, \ldots, z_{m}+b\right)=(y+b) \otimes(u+b) \\
= & {\left[\left(y+b+c_{23}\right)\left(a_{1}+a_{3}+b\right)+\left(u+b+c_{13}\right)\left(a_{2}+a_{3}+b\right)\right]\left(a_{1}+a_{2}+b\right) } \\
= & {\left[\left(y+b_{1}+b_{2}+b_{3}+c_{23}\right)\left(a_{1}+a_{3}\right)\right.} \\
& \left.\quad+\left(u+b_{1}+b_{2}+b_{3}+c_{13}\right)\left(a_{2}+a_{3}\right)+b\right]\left(a_{1}+a_{2}\right)+b \\
= & {\left[\left(y+c_{23}\right)\left(a_{1}+a_{3}\right)+\left(u+c_{13}\right)\left(a_{2}+a_{3}\right)+b_{1}+b_{2}+b_{3}\right]\left(a_{1}+a_{2}\right)+b } \\
= & {\left[\left(y+c_{23}\right)\left(a_{1}+a_{3}\right)+\left(u+c_{13}\right)\left(a_{2}+a_{3}\right)\right]\left(a_{1}+a_{2}\right)+b } \\
= & (y \otimes u)+b=w+b .
\end{aligned}
$$

2. Unsolvability. Let $\mathrm{FG}\left(x_{1}, \ldots, x_{n}\right)$ and $\mathrm{FG}\left(y_{1}, \ldots, y_{n}\right)$ be free groups. Let $A$ be a finitely presented group with unsolvable word problem. Say $A$ is generated by $x_{1}, \ldots, x_{n}$ subject to the relations $u_{1}=u_{2}=\ldots=u_{m}=1$, where $u_{1}, \ldots, u_{m}$ are group-theoretic terms (words) in $x_{1}, \ldots, x_{n}$. Let $N$ be the normal subgroup of $\mathrm{FG}\left(x_{1}, \ldots, x_{n}\right)$ generated by $u_{1}, \ldots, u_{m}$, so that $A \cong \mathrm{FG}\left(x_{1}, \ldots, x_{n}\right) / N$. Let $G=\mathrm{FG}\left(x_{1}, \ldots, x_{n}\right) \times \mathrm{FG}\left(y_{1}, \ldots, y_{n}\right)$ and let $H$ be the subgroup of $G$ generated by $x_{i} y_{i}, i=1, \ldots, n$, and $u_{j}, j=1, \ldots, m$.

LEMMA 2.1. $N \subseteq H$.

Proof. Since the $y_{i}$ commute with the $x_{k}$, and hence with the $u_{j}, x_{i}^{-1} u_{j} x_{i}=$ $\left(x_{i} y_{i}\right)^{-1} u_{j}\left(x_{i} y_{i}\right) \in H$.

Actually $N=\mathrm{FG}\left(x_{1}, \ldots, x_{n}\right) \cap H$, as is shown in Lemma 6.5.1 of [3].

LEMMA 2.2. There is a skew field $D$ such that $G$ is a subgroup of the multiplicative group of $D$. Moreover there is a $t \in D$ such that for $w \in \mathrm{FG}\left(x_{1}, \ldots, x_{n}\right), w \in N$, if and only if $t w=w t$ in $D$. Also $D$ satisfies the following.

$$
\begin{aligned}
& x_{i} y_{j}=y_{j} x_{i}, \quad i, j=1, \ldots, n, \\
& t x_{i} y_{i}=x_{i} y_{i} t, \quad i=1, \ldots, n, \\
& t u_{j}=u_{j} t, \quad j=1, \ldots, m .
\end{aligned}
$$

Proof. This is Lemma 6.5.2 of [3]. Our $D$ is Cohn's $D(t ; \sigma)$. We shall assume that $D$ is generated by $x_{1}, \ldots, x_{n}, y_{1}, \ldots, y_{n}, t$. If this were not the case we could simply take the subfield generated by these elements. Thus $D$ is countably infinite.

Let $L\left(D^{4}\right)$ be the lattice of subspaces of $D^{4}$ as a left vector space over $D$. Let $a_{1}=D(1,0,0,0), \ldots, \quad a_{4}=D(0,0,0,1), c_{12}=D(-1,1,0,0), \ldots, c_{14}=$ $D(-1,0,0,1)$. It is easy to see that $\left\{a_{1}, a_{2}, a_{3}, a_{4}, c_{12}, c_{13}, c_{14}\right\}$ is a 4-frame in 
$L\left(D^{4}\right)$. Using elementary linear algebra it is easy to see that a subspace of $D^{4}$ is a complement of $a_{2}$ in $a_{1}+a_{2} / 0$ if and only if it has the form $D(-1, d, 0,0)$, and moreover for $d^{\prime} \neq d, D\left(-1, d^{\prime}, 0,0\right) \neq D(-1, d, 0,0)$. Furthermore, $D(-1, d, 0,0) \oplus D\left(-1, d^{\prime}, 0,0\right)=D\left(-1, d+d^{\prime}, 0,0\right)$ and $D(-1, d, 0,0) \otimes$ $D\left(-1, d^{\prime}, 0,0\right)=D\left(-1, d d^{\prime}, 0,0\right)$, where $\oplus$ and $\otimes$ are defined by (1.6) and (1.7). Thus the ring associated with the above frame is isomorphic to $D$ and we will identify each $d$ in $D$ with $D(-1, d, 0,0)$.

Let $q$ be a prime greater than $2 n+2$ and $K$ a countably infinite field of characteristic $q$. Let $L\left(K^{4}\right)$ be the lattice of subspaces of $K^{4}$ as a vector space over $K$. Let $a_{1}^{\prime}=K(1,0,0,0), \ldots, a_{4}^{\prime}=K(0,0,0,1), c_{12}^{\prime}=K(-1,1,0,0), \ldots, c_{14}^{\prime}=$ $K(-1,0,0,1)$. Let $0^{\prime}$ be the least element of $L\left(K^{4}\right)$. The quotient sublattices $a_{1}+a_{2} / 0$ in $L\left(D^{4}\right)$ and $a_{1}^{\prime}+a_{2}^{\prime} / 0^{\prime}$ in $L\left(K^{4}\right)$ are both isomorphic to $M_{\omega}$ (the countably infinite two-dimensional lattice). Let $\sigma: a_{1}+a_{2} / 0 \rightarrow a_{1}^{\prime}+a_{2}^{\prime} / 0^{\prime}$ be a lattice isomorphism such that

$$
\begin{aligned}
& \sigma\left(a_{1}\right)=a_{1}^{\prime}, \quad \sigma\left(a_{2}\right)=a_{2}^{\prime}, \quad \sigma\left(c_{12}\right)=c_{12}^{\prime}, \quad \sigma\left(x_{i}\right)=\alpha_{12}^{i+1}\left(a_{1}^{\prime}\right), \\
& \sigma\left(y_{i}\right)=\alpha_{12}^{n+1+i}\left(a_{1}^{\prime}\right), \quad \sigma(t)=\alpha_{12}^{2 n+2}\left(a_{1}^{\prime}\right) .
\end{aligned}
$$

Recall that $x_{i}=D\left(-1, x_{i}, 0,0\right)$, etc. We let $\alpha_{12}\left(a_{1}^{\prime}\right)$ be the element obtained from (1.8) using the frame $\left\{a_{i}^{\prime}, c_{1 i}^{\prime}\right\}$. This convention of letting the argument signify in which frame $\alpha_{12}$ is to be evaluated will be used below. Note that $\alpha_{12}^{r}\left(a_{1}^{\prime}\right)=$ $K(-1, r, 0,0)$ for $r \in Z$.

Let $L$ be the lattice on the disjoint union of $L\left(D^{4}\right)$ and $L\left(K^{4}\right)$ whose ordering is the transitive closure of the orderings on $L\left(D^{4}\right)$ and $L\left(K^{4}\right)$, and the ordering $z+a_{3}+a_{4}<\sigma(z), z \in a_{1}+a_{2} / 0$. This is essentially the Hall-Dilworth gluing of the dual ideal $1 / a_{3}+a_{4}$ of $L\left(D^{4}\right)$ to the ideal $a_{1}^{\prime}+a_{2}^{\prime} / 0^{\prime}$ of $L\left(K^{4}\right)$ via $\sigma$. We have kept the intervals $1 / a_{3}+a_{4}$ and $a_{1}^{\prime}+a_{2}^{\prime} / 0^{\prime}$ separate, since this is more convenient for our purposes. Thus $L$ is actually the Hall-Dilworth sum of $L\left(D^{4}\right), M_{\omega} \times 2$, and $L\left(K^{4}\right)$ glued over two-dimensional quotients. $L$ is modular by [8] and [18]. The sublattice of $L$ generated by $\left\{a_{i}, a_{i}^{\prime}\right\}$ is diagrammed in Figure 1 of [14]. Note that $L\left(D^{4}\right)$ and $L\left(K^{4}\right)$ are both sublattices of $L$ and that in $L a_{1}+a_{2} / 0 \nearrow a_{1}^{\prime}+a_{2}^{\prime} / 0^{\prime}$ and the induced isomorphism, $z \rightarrow z+0^{\prime}$, agrees with $\sigma$. The inverse isomorphism is $z^{\prime} \rightarrow z^{\prime}\left(a_{1}+a_{2}\right)$, of course. Hence $x_{i}=\left(\alpha_{12}^{i+1}\left(a_{1}^{\prime}\right)\right)\left(a_{1}+a_{2}\right)$ and similar formulae hold for $y_{i}$ and $t$. Thus all the elements we are interested in lie in the sublattice of $L$ generated by $\left\{a_{i}, a_{i}^{\prime}, c_{1 j}, c_{1 j}^{\prime}\right\}$. It is shown in Theorem 3.4 of [14] that this sublattice is in fact generated by the five elements $a_{3}^{\prime}+a_{4}^{\prime}, c_{23}^{\prime}, c_{13}^{\prime}+c_{24}^{\prime}, a_{1}+a_{2}$ and $c_{13}+c_{24}$. We may assume $L$ is generated by these five elements. Let $f$ be a homomorphism of FM(5) onto $L$. As is shown in the proof of Theorem 2.1 of [14], it is possible to effectively find $\mathbf{a}_{i}, \mathbf{c}_{1 j}, \mathbf{a}_{i}^{\prime}, \mathbf{c}_{1 j}^{\prime} \in \mathrm{FM}(5)$ such that the following hold.

$$
f\left(\mathbf{a}_{i}\right)=a_{i}, \quad f\left(\mathbf{a}_{i}^{\prime}\right)=a_{i}^{\prime}, \quad f\left(\mathbf{c}_{1 j}\right)=c_{1 j}, \quad f\left(\mathbf{c}_{1 j}^{\prime}\right)=c_{1 j}^{\prime} ;
$$

$\left\{\mathbf{a}_{i}, \mathbf{c}_{1 j}\right\}$ is a 4-frame and $\left\{\mathbf{a}_{i}^{\prime}, \mathbf{c}_{1 j}^{\prime}\right\}$ is a 4-frame of characteristic $q$;

$$
\begin{gathered}
a_{1}+a_{2} / 0 \nearrow a_{1}+a_{2}+a_{3}+a_{4} / a_{3}+a_{4} \nearrow a_{1}^{\prime}+a_{2}^{\prime} / 0^{\prime}, \\
a_{i}+0^{\prime}=a_{i}^{\prime}, \quad i=1,2, \quad c_{12}+0^{\prime}=c_{12}^{\prime} .
\end{gathered}
$$


Whenever we have elements $\mathbf{a}_{i}, \mathbf{c}_{1 j}, \mathbf{a}_{i}^{\prime}, \mathbf{c}_{1 j}^{\prime}$ in $\operatorname{FM(5)~satisfying~the~above~we~let~}$

$$
\begin{gathered}
\mathbf{x}_{i}=\alpha_{12}^{i+1}\left(\mathbf{a}_{1}^{\prime}\right)\left(\mathbf{a}_{1}+\mathbf{a}_{2}\right), \quad \mathbf{y}_{i}=\alpha_{12}^{n+1+i}\left(\mathbf{a}_{1}^{\prime}\right)\left(\mathbf{a}_{1}+\mathbf{a}_{2}\right), \quad i=1, \ldots, n, \\
\mathbf{t}=\alpha_{12}^{2 n+2}\left(\mathbf{a}_{1}^{\prime}\right)\left(\mathbf{a}_{1}+\mathbf{a}_{2}\right) .
\end{gathered}
$$

Observe that $f\left(\mathbf{x}_{i}\right)=x_{i}, f\left(\mathbf{y}_{i}\right)=y_{i}$ and $f(\mathbf{t})=t$.

Let $\mathbf{R}$ be the ring associated with the frame $\left\{a_{i}, \mathbf{c}_{1 j}\right\}$ (see (1.5)). Since $\alpha_{12}^{r}\left(a_{i}^{\prime}\right)$ is a relative complement of $a_{2}^{\prime}$ in $a_{1}^{\prime}+a_{2}^{\prime} / 0^{\prime}$ (by Lemma 1.4 of [14]), $x_{i}, y_{i}$ and $t$ are all in $\mathbf{R}$. If $0<r<q$ then $\alpha_{12}^{r}\left(\mathbf{a}_{1}^{\prime}\right)$ corresponds to $r$ in the ring associated with the frame $\left\{\mathbf{a}_{i}^{\prime}, \mathbf{c}_{1 j}^{\prime}\right\}$. But this ring has characteristic $q$ since the frame does. Thus, by Lemma 1.2, $\alpha_{12}^{r}\left(a_{1}^{\prime}\right)$ is relative complement of $a_{1}^{\prime}$ in $a_{1}^{\prime}+a_{2}^{\prime} / 0^{\prime}$. Hence $x_{i}, y_{i}$, and $t$ are all complements of $a_{1}$ in $a_{1}+a_{2} / 0$. Hence $x_{i}, y_{i}$ and $t$ are invertible elements of R. Our goal is to adjust the choice of $\left\{\mathbf{a}_{i}, \mathbf{a}_{i}^{\prime}, \mathbf{c}_{1 j}, \mathbf{c}_{1 j}^{\prime}\right\}$ so that (2.5), (2.6), (2.7) still hold and moreover the relations (2.1), (2.2), (2.3) also hold in $\mathbf{R}$.

Suppose $b_{2} \in \operatorname{FM}(5)$ with $0 \leqslant b_{2} \leqslant a_{2}$. Then we let $b_{1}=\left(b_{2}+c_{12}\right) a_{1}, b_{i}=$ $\left(b_{1}+c_{1 i}\right) a_{i}, i=3,4$, and $b=b_{1}+\cdots+b_{4}$. Also we let $b_{1}^{\prime}=b_{1}+0^{\prime}$ and $b_{i}^{\prime}=$ $\left(b_{1}^{\prime}+c_{1 i}^{\prime}\right) a_{i}^{\prime}, i=2,3,4, b^{\prime}=b_{1}^{\prime}+\cdots+b_{4}^{\prime}$. Observe that $b_{2}^{\prime}=b_{2}+0^{\prime}$ because $c_{12}^{\prime}=$ $\mathbf{c}_{12}+\mathbf{0}^{\prime}$. Also observe that if $\left\{\mathbf{a}_{i}, \mathbf{c}_{1 j}, \mathbf{a}_{i}^{\prime}, \mathbf{c}_{1 j}^{\prime}\right\}$ satisfies (2.5), (2.6), (2.7) then $\left\{\mathbf{a}_{i}+\mathbf{b}\right.$, $\left.\mathbf{c}_{1 j}+\mathbf{b}, \mathbf{a}_{i}^{\prime}+\mathbf{b}^{\prime}, \mathbf{c}_{1 j}^{\prime}+\mathbf{b}^{\prime}\right\}$ also satisfies (2.5), (2.6), (2.7) provided that $f\left(\mathbf{b}_{i}\right)=0$ for one, and hence all, $i$. Let $\mathbf{R}(\mathbf{b})$ be the ring associated with the frame $\left\{a_{i}+\mathbf{b}, \mathbf{c}_{1 j}+\right.$ b) and let $x_{i}(b)=\left[\alpha_{12}^{i+1}\left(a_{1}^{\prime}+b^{\prime}\right)\right]\left(a_{1}+a_{2}+b\right)$, i.e. $x_{i}(b)$ is the $x_{i}$ associated with the new frame. (Recall that $\alpha_{12}\left(a_{1}^{\prime}+b^{\prime}\right)$ is obtained from (1.8) by replacing $a_{i}$ by $\mathbf{a}_{i}^{\prime}+\mathbf{b}^{\prime}$ and $\mathbf{c}_{i j}$ by $\mathbf{c}_{i j}^{\prime}+\mathbf{b}^{\prime}$.) The elements $\mathbf{y}_{i}(\mathbf{b})$ and $\mathbf{t}(\mathbf{b}) \in \mathbf{R}(\mathbf{b})$ are defined in $\mathbf{a}$ similar manner.

LEMMA 2.3. $\mathbf{x}_{i}(\mathbf{b})=\mathbf{x}_{i}+$ b. Similar formulae hold for $\mathbf{y}_{i}(\mathbf{b})$ and $\mathbf{t}(\mathbf{b})$.

Proof. By definition $x_{i}=\alpha_{12}^{i+1}\left(a_{1}^{\prime}\right)\left(a_{1}+a_{2}\right)$. Thus

$$
x_{i}+0^{\prime}=\alpha_{12}^{i+1}\left(a_{1}^{\prime}\right)\left(a_{1}+a_{2}+0^{\prime}\right)=\alpha_{12}^{i+1}\left(a_{1}^{\prime}\right)\left(a_{1}^{\prime}+a_{2}^{\prime}\right)=\alpha_{12}^{i+1}\left(a_{1}^{\prime}\right)
$$

Since $b_{i}^{\prime}=b_{i}+0^{\prime}, i=1,2$, and since $\alpha_{12}^{i+1}\left(a_{1}^{\prime}+b^{\prime}\right)=\alpha_{12}^{i+1}\left(a_{1}^{\prime}\right)+b^{\prime}$ by Lemma 1.2 of [14], we have

$$
\begin{aligned}
\mathbf{x}_{i}(\mathbf{b}) & =\alpha_{12}^{i+1}\left(\mathbf{a}_{1}^{\prime}+\mathbf{b}^{\prime}\right)\left(\mathbf{a}_{1}+\mathbf{a}_{2}+\mathbf{b}\right) \\
& =\left(\alpha_{12}^{i+1}\left(\mathbf{a}_{1}^{\prime}\right)+\mathbf{b}^{\prime}\right)\left(\mathbf{a}_{1}+\mathbf{a}_{2}\right)+\mathbf{b} \\
& =\mathbf{b}+\left(\alpha_{12}^{i+1}\left(\mathbf{a}_{1}^{\prime}\right)+\mathbf{b}^{\prime}\right)\left(\mathbf{a}_{1}^{\prime}+\mathbf{a}_{2}^{\prime}\right)\left(\mathbf{a}_{1}+\mathbf{a}_{2}\right) \\
& =\mathbf{b}+\left(\alpha_{12}^{i+1}\left(\mathbf{a}_{1}^{\prime}\right)+\mathbf{b}_{1}^{\prime}+\mathbf{b}_{2}^{\prime}\right)\left(\mathbf{a}_{1}+\mathbf{a}_{2}\right) \\
& =\mathbf{b}+\left(\mathbf{b}_{1}+\mathbf{b}_{2}+\mathbf{x}_{i}+\mathbf{0}^{\prime}\right)\left(\mathbf{a}_{1}+\mathbf{a}_{2}\right) \\
& =\mathbf{b}+\mathbf{b}_{1}+\mathbf{b}_{2}+\mathbf{x}_{i}=\mathbf{b}+\mathbf{x}_{i} .
\end{aligned}
$$

From Lemma 1.4 we have $\alpha_{12}^{i+1}\left(a_{1}^{\prime}\right)+b_{1}^{\prime}=: \alpha^{i+1}\left(a_{1}^{\prime}\right)+b_{2}^{\prime}$ and from this it easily follows that $\mathbf{x}_{i}+\mathbf{b}_{1}=\mathbf{x}_{i}+\mathbf{b}_{2}, i=1, \ldots, n$, and similarly for $\mathbf{y}_{i}$ and $\mathbf{t}$. Combining this and Lemmas 1.6 and 2.3 we have the following.

LeMMA 2.4. Let $w$ be a group theoretic term in $2 n+1$ variables and let $w$ be the interpretation of $w$ in $\mathbf{R}$ using $\mathbf{x}_{1}, \ldots, \mathbf{x}_{n}, \mathbf{y}_{1}, \ldots, \mathbf{y}_{n}$, t. Let $\mathbf{w}(\mathbf{b})$ be the corresponding element of $\mathbf{R}(\mathbf{b})$ using $\mathbf{x}_{1}(\mathbf{b}), \ldots, \mathbf{x}_{n}(\mathbf{b}), \mathbf{y}_{1}(\mathbf{b}), \ldots, \mathbf{y}_{n}(\mathbf{b}), \mathbf{t}(\mathbf{b})$ (and, of course, multiplication in $\mathbf{R}(\mathbf{b}))$. Then $\mathbf{w}(\mathbf{b})=\mathbf{w}+\mathbf{b}$. 
With the aid of the previous lemma it is easy to adjust the frames so that the relations (2.1) (2.2) (2.3) hold in $\mathbf{R}$. In order to make $\mathbf{x}_{1}$ and $\mathbf{y}_{1}$ commute, for example, set $b_{2}=\left(x_{1} \otimes y_{1}+y_{1} \otimes x_{1}\right) a_{2}$. Define the $b_{i}$ and $b$ in the usual way. Then in $R(b)$ we have, since $b \geqslant b_{2}$ and $x_{1} \otimes y_{1} \in R$,

$$
\begin{aligned}
& x_{1}(b) \otimes_{b} y_{1}(b)=x_{1} \otimes y_{1}+b+\left(x_{1} \otimes y_{1}+y_{1} \otimes x_{1}\right) a_{2} \\
& =b+\left(x_{1} \otimes y_{1}+y_{1} \otimes x_{1}\right)\left(x_{1} \otimes y_{1}+a_{2}\right) \\
& =b+\left(x_{1} \otimes y_{1}+y_{1} \otimes x_{1}\right)\left(a_{1}+a_{2}\right) \\
& =b+x_{1} \otimes y_{1}+y_{1} \otimes x_{1} \text {. }
\end{aligned}
$$

Similarly $\mathbf{y}_{1}(b) \otimes_{b} x_{1}(b)=b+x_{1} \otimes y_{1}+y_{1} \otimes x_{1}$. Hence $x_{1}(b) \otimes_{b} y_{1}(b)=y_{1}(b)$ $\otimes_{b} \mathbf{x}_{1}(\mathbf{b})$.

Also note that, by Lemma 2.4, if one of the relations of (2.1)-(2.3) already holds in $\mathbf{R}$ it will always hold in $\mathbf{R}(\mathbf{b})$ (for any choice of b). Thus after relabelling we obtain the following.

LemMA 2.5. There exist elements $\mathbf{a}_{i}, \mathbf{a}_{i}^{\prime}, \mathbf{c}_{1 j}, \mathbf{c}_{1 j}^{\prime} \in \operatorname{FM}(5), i=1,2,3,4, j=2,3,4$, such that (2.5), (2.6), (2.7) hold and the elements $\mathbf{x}_{i}, \mathbf{y}_{i}, \mathbf{t}, i=1, \ldots, n$, defined by (2.8) satisfy the relations (2.1), (2.2), (2.3) in $\mathbf{R}$.

THEOREM 2.6. The word problem for $\operatorname{FM}(5)$ is unsolvable.

Proof. Let $G$ be the subgroup of $(\mathbf{R}, \otimes)$ generated by $\mathbf{x}_{1}, \ldots, \mathbf{x}_{n}, \mathbf{y}_{1}, \ldots, \mathbf{y}_{n}$. Since the operation $\otimes$ in $\mathbf{R}$ is defined in terms of the lattice operations, the map $f$ restricted to $\mathbf{G}$ is a group homomorphism from $\mathbf{G}$ onto $\boldsymbol{G}$. But $\mathbf{G}$ satisfies (2.1) which are the defining relations of the group $\mathrm{FG}\left(x_{1}, \ldots, x_{n}\right) \times \mathrm{FG}\left(y_{1}, \ldots, y_{n}\right)=$ $G$. Hence $\left.f\right|_{\mathbf{G}}: \mathbf{G} \rightarrow G$ is an isomorphism of groups. Let $w$ be a group-theoretic term in $x_{1}, \ldots, x_{n}$ and let $\mathbf{w}$ be the corresponding element in $\mathbf{G}$. We claim $\mathbf{w} \in \mathbf{N}$ (the normal subgroup of $\mathbf{G}$ corresponding to $N$ in $G$ ) if and only if $\mathbf{w} \otimes \mathbf{t}=\mathbf{t} \otimes \mathbf{w}$ holds in $\mathbf{R}$. Firstly, if $\mathbf{w} \in \mathbf{N}$ then $\mathbf{w} \in \mathbf{H}$ by Lemma 2.1 and $\mathbf{t}$ commutes with everything in $\mathbf{H}$ by (2.2) and (2.3). Conversely if $\mathbf{w} \otimes \mathbf{t}=\mathbf{t} \otimes \mathbf{w}$ then by applying $f$ we get $w t=t w$ in $D$ which implies $w \in N \subseteq D$. Since $f$ is an isomorphism on $G$, this implies $\mathbf{w} \in \mathbf{N}$. From this it easily follows that if the word problem for FM(5) were solvable then the word problem for $A \cong \mathrm{FG}\left(x_{1}, \ldots, x_{n}\right) / N$ would be solvable, a contradiction.

Notice that for $\mathbf{w} \in \mathbf{G}, \mathbf{w} \otimes \mathbf{t}=\mathbf{t} \otimes \mathbf{w}$ holds if and only if $\mathbf{w} \otimes \mathbf{t}-\mathbf{t} \otimes \mathbf{w}=\mathbf{0}_{\mathbf{R}}$ $=a_{1}$ which holds if and only if $t=w^{-1} \otimes t \otimes w$. Hence if we choose $u_{0}=a_{0}$ or $u_{0}=\mathrm{t}$, there is no recursive procedure to determine if $v=u_{0}$ holds in all modular lattices for five variable lattice terms $v$.

3. Discussion. We constructed the lattice $L$ in such a way that the elements $x_{i}, y_{i}$ and $t$ in $D$ were lined up with nonzero elements of the prime subfield of $K$. This served two purposes in the proof. First it allowed us to find inverse images $x_{i}$ of $x_{i}$, etc., in $\mathbf{R}$ and secondly it permitted us to make corrections in our frame and have the new inverse images of the $x_{i}$ behave correctly with respect to the old ones (see Lemma 2.3). It is natural to ask if this use of $K$ is really necessary. That is, would it have been possible to prove the unsolvability results by simply considering the 
homomorphism from FM(5) onto $L\left(D^{4}\right)$ ? The answer is no. If the answer were yes then we would have as a corollary to the proof that $F_{\mathfrak{r}}(5)$ has an unsolvable word problem for any variety of modular lattices $\mathcal{V}$ containing $L\left(D^{4}\right)$. In particular for the variety $Q$ generated by all subgroup lattices of abelian groups $F_{\mathbb{Q}}(5)$ would have an unsolvable word problem. However, by a theorem of Herrmann and Huhn [22], [19], this is not the case.

The situation for word problems in varieties of modular lattices is this: if $\mathcal{V}$ is a variety of modular lattices containing our lattice $L$, then $F_{\gamma}(5)$ has an unsolvable word problem. These varieties include the variety generated by all modular lattices of breadth at most $n, n \geqslant 4$, and also the variety of all $n$-distributive lattices, for $n \geqslant 4$. However, $L$ is nonarguesian [27] and thus the following question is open: do the free arguesian lattices have solvable word problems? Of course, by the results of Herrmann and Huhn cited above, the word problem for free lattices in certain nice varieties is solvable (see also [26]). Perhaps a more important problem is this: does FM(4) have a solvable word problem?

\section{REFERENCES}

1. B. Artmann, On coordinates in modular lattices with a homogeneous basis, Illinois J. Math. 12 (1968), 626-648.

2. G. Birkhoff, Lattice theory, Amer. Math. Soc. Colloq. Publ., vol. 25, rev. ed., Amer. Math. Soc., New York, 1948.

3. P. M. Cohn, Skew field constructions, London Math. Soc. Lecture Note Series, no. 27, Cambridge Univ. Press, Cambridge, 1977.

4. P. Crawley and R. P. Dilworth, Algebraic theory of lattices, Prentice-Hall, Englewood Cliffs, N. J., 1973.

5. M. Davis, Computability and unsolvability, McGraw-Hill, New York, 1958.

6. A. Day, C. Herrman and R. Wille, On modular lattices with four generators, Algebra Universalis 2 (1972), 317-323.

7. R. Dedekind, Uber die drei Moduln erzeugte Dualgruppe, Math. Ann. 53 (1900), 371-403.

8. R. P. Dilworth, The arithmetical theory of Birkhoff lattices, Duke Math. J. 8 (1941), 286-299.

9. R. P. Dilworth and R. Freese, Generators for lattice varieties, Algebra Universalis 6 (1976), 263-267.

10. T. Evans, Word problems, Bull. Amer. Math. Soc. 84 (1978), 789-802.

11. R. Freese, Breadth two modular lattices, Proc. Univ. Houston Lattice Theory Conf. (Houston, Texas, 1973), Dept. Math., Univ. Houston, Houston, Texas, 1973, pp. 409-451.

12. __ Planar sublattices of FM(4), Algebra Universalis 6 (1976), 69-72.

13. Some varieties of modular lattices not generated by their finite dimensional members, Contribution to Universal Algebra, Szeged, Hungary, Colloq. Math. Soc. Janos Bolyai 17 (1977), 133-144.

14. The variety of modular lattices is not generated by its finite members, Trans. Amer. Math. Soc. 255 (1979), 277-300.

15. __ Projective geometries as projective modular lattices, Trans. Amer. Math. Soc. 251 (1979), $329-342$.

16. __ Free modular lattices, Notices Amer. Math. Soc. 26 (1979), \# 79T-A4.

17. M. M. Gluhov, Algorithmic solvability of the word problem for completely free modular lattices, Sibirsk. Math. Ż. 5 (1964), $1027-1034$.

18. M. Hall and R. P. Dilworth, The imbedding problem for modular lattices, Ann. of Math. (2) 45 (1944), 450-456.

19. C. Herrmann, On the equational theory of submodule lattices, Proc. Univ. Houston Lattice Theory Conf. (Houston, Texas, 1973), Dept. Math., Univ. Houston, Houston, Texas, 1973, pp. 105-118.

20. Concerning M. M. Gluhov's paper on the word problem for free modular lattices, Algebra Universalis 5 (1975), 445. 
21. , On modular lattices generated by two complemented pairs, Houston J. Math. 2 (1976), 513-523.

22. C. Herrmann and A. Huhn, Zum Wortproblem fur freie Untermodulverbande, Arch. Math. 26 (1975), 449-453.

23. __ Lattices of normal subgroups which are generated by frames, Lattice Theory, Szeged, Hungary, Colloq. Math. Soc. Janos Bolyai 14 (1975), 97-136.

24. G. Hutchinson, Recursively unsolvable word problems for modular lattices and diagram-chasing, J. Algebra 26 (1973), 385-399.

25. E__ Embedding and unsolvability theorems for modular lattices, Algebra Universalis 7 (1977), 47-84.

26. G. Hutchinson and G. Czedli, A test for identities satisfied in lattices of submodules, Algebra Universalis 8 (1978), 269-309.

27. B. Jónsson, Arguesian lattices of dimension $n<4$, Math. Scand. 7 (1959), 133-145.

28. __ Varieties of lattices: some open problems, Colloq. Math. Soc. J. Bolyai, Universal Algebra Conference, Esztergom, Hungary, 1977.

29. A. Macintyre, The word problem for division rings, J. Symbolic Logic 38 (1973), 428-436.

30. F. Maeda, Kontinuierliche Geometrien, Springer-Verlag, Berlin, 1958.

31. J. von Neumann, Continuous geometry, (I. Halperin, ed.), Princeton Univ. Press, Princeton, N. J., 1960.

32. G. Sauer, W. Seibert and R. Wille, On free modular lattices over partial lattices with four generators, Proc. Univ. Houston Lattice Theory Conf. (Houston, Texas, 1973), Dept. Math., Univ. Houston, Houston, Texas, 1973, pp. 332-382.

33. M. Schutzenberger, Le probleme des mots dans les treillis modulaires libres, C. R. Acad. Sci. Paris Sér. A-B 237 (1953), 507-508.

34. K. Takeuchi, On free modular lattices, Japan J. Math. 21 (1951), 53-65.

35. __ On free modular lattices. II, Tôhoku Math. J. 11 (1959), 1-12.

36. P. Whitman, Free lattices, Ann. of Math. (2) 42 (1941), 325-330.

37. Free lattices. II, Ann. of Math. (2) 43 (1942), 104-115.

38. _ review of [33], Math. Rev. 15 (1954), 192.

39. __ Status of word problems for lattices, Proc. Sympos. Pure Math., Vol. 2, Amer. Math. Soc., Providence, R. I., 1961, pp. 17-21.

40. R. Wille, Über modulare Verbande die von einer endlichen halbgeordneten Menge frei erzeugt werden, Math. Z. 131 (1973), 241-249.

41. L. Lipshitz, The undecidability of the word problems for projective geometries and modular lattices, Trans. Amer. Math. Soc. 193 (1974), 171-180.

Department of Mathematics, University of HawaII, Honolulu, Hawail 96822 (Current address)

Department of Mathematics, Vanderbilt University, Nashville, TenNessee 37203 